\title{
Signaling mechanisms
}

\author{
Michael D. Ehlers and \\ Department of Neurobiology, Howard Hughes Medical Institute, Duke University Medical Center, \\ Box 3209, Durham, North Carolina, 27701, USA \\ Gina Turrigiano \\ Department of Biology MS08, Brandeis University, 415 South St, Waltham, MA 02454
}

Michael D. Ehlers: ehlers@neuro.duke.edu; Gina Turrigiano:

\section{Introduction}

Uncovering cell signaling mechanisms is requisite for understanding nearly all facets of brain development and function. This issue of Current Opinion in Neurobiology contains 17 articles that discuss a number of burning issues in the field, ranging from the role of semaphorin signaling, to mechanisms of synapse development, to assembly of visual circuits. This diversity of subjects reflects the broad importance of signaling mechanisms throughout neuroscience and the rapid progress that is being made on multiple fronts. This collection of reviews highlights the excitement and fusion of neuroscience across traditional boundaries of molecular mechanism and systems analysis. We discuss below some of the fundamental advances covered in this collection of reviews, and their wider implications.

\section{Signaling complexes and synaptic plasticity}

Intracellular signaling pathways are best thought of as signaling webs, with extensive crosstalk between classical pathways. Several reviews in this issue highlight the complexity of these signaling pathways and their effects on neuronal function. Calcium channels are critical for many aspects of neuronal function, including presynaptic neurotransmitter release, and various forms of intrinsic and synaptic plasticity. In the review by Dolphin, a novel way of modulating calcium channel function through association with beta subunits is discussed. De Jong and Verhage describe the many calcium binding proteins in the presynaptic terminal, and some of the pathways that allow this calcium sensing to modulate neurotransmitter release. In addition to short-term effects on transmitter release, signaling back to the presynaptic terminal from the postsynaptic neuron can induce a number of forms of long-lasting synaptic plasticity, and recent advances in understanding how plasticity at inhibitory synapses is controlled by such signaling is described in the review by Kauer and McBain.

\section{Intercellular signaling across the lifespan}

Many forms of neural signaling arise during early brain development to pattern the growth of axons and the formation of synapses. Beyond development, these same signaling molecules persist in more mature nervous systems where they modify synaptic strength and contribute to plasticity in the adult brain. The review by Pasterkamp and Giger summarizes recent results on semaphorins, well-known mediators of axon guidance during development, and their novel

\footnotetext{
Publisher's Disclaimer: This is a PDF file of an unedited manuscript that has been accepted for publication. As a service to our customers we are providing this early version of the manuscript. The manuscript will undergo copyediting, typesetting, and review of the resulting proof before it is published in its final citable form. Please note that during the production process errors may be discovered which could affect the content, and all legal disclaimers that apply to the journal pertain.
} 
roles in regulating synapse structure, physiology, and plasticity in the mature brain. Another example of signaling systems prominent during brain development yet abundant in the adult are the receptor tyrosine kinases of the Eph family and their membrane-bound ligands the ephrins. In their article, Lai and Ip review our current understanding of how ephrins and Eph receptors, originally identified as regulators or axon guidance and topographic mapping, participate in synapse formation and synaptic plasticity.

\section{Signaling through circuits}

Ultimately, signaling mechanisms construct circuits through which patterns of activity produce brain function. Direction-selective neurons in visual systems which respond preferentially to motion in one direction are a particularlly amazing and long-studied example of signaling specificity within a sensory circuit. In their article, Elstrott and Feller review recent advances revealing fundamental differences in the establishment of direction-selective circuits in the retina and visual cortex. Visual cortical plasticity mechanisms are proving to be far more diverse than originally thought, and clearly encompass Hebbian, homeostatic, and inhibitory plasticity mechanisms that cooperate to generate functional circuits. In their review, McCoy, Huang, and Philpot discuss some of the recent advances in understanding the activitydependent signaling mechanisms that contribute to and modulate this circuit refinement. The function of all circuits is shaped by GABAergic inhibition. GABA is the main inhibitory neurotransmitter in the mammalian brain, and perturbed GABA signaling is the underlying cause of many neuropsychiatric disorders. The review by Koch and Magnusson discusses new forms of GABA signalling that are associated with unconventional release of GABA, and review the functional consequences of such GABA signaling in the mature brain.

\section{Signaling to build and modify synapses}

The paramount structure for neuronal signaling is the synapse, whose molecular components must be precisely assembled and whose properties tightly tuned to accommodate information transfer. In this issue, Owald and Sigrist explain the assembly process of the presynaptic active zone, the macromolecular ensemble that controls presynaptic neurotransmitter release. Once assembled, local activity-dependent synthesis of new proteins has long been proposed to execute long-lasting input-specific changes at the synapse. Waung and Huber describe recent studies on group 1 metabotropic glutamate receptor-dependent long-term depression (mGluRLTD) that have provided important insight into mechanisms of local dendritic translation and plasticity, and its dysfunction in one of the most common genetic forms of human mental retardation, Fragile $\mathrm{X}$ syndrome. In addition to input-specific modulation of synaptic transmission, there are also homeostatic mechanisms that can regulate neuronal excitability and synaptic strength either globally, or in particular neuronal compartments. In their review, Yu and Goda discuss recent advances in our understanding of the diversity of such homeostatic mechanisms, and in particular the role of dendritic signaling in generating local changes that are confined to particular dendritic compartments. Their review highlights the notion that the signaling mechanisms underlying neuronal plasticity can operate over a range of spatial scales.

\section{Neuronal signaling to the nucleus}

Intracellular signals influence the pattern of gene expression, thereby controlling diverse aspects of neuronal fate and function. Recent data indicate that epigenetic mechanisms play an important role in regulating synaptic plasticity, learning, and memory. Roth and Sweatt discuss how post-translational histone modifications and DNA methylation are modified by neuronal activity and may prime patterns of gene expression that encode memory states. 


\section{Conclusions}

In putting together this issue, we have cast a wide net across the ocean of signaling mechanisms that contribute to brain function. We have deliberately assembled topics that range from intracellular signaling within individual neurons to signaling mechanisms of plasticity and circuit function. We have attempted to address signaling mechanisms that span temporal domains from rapid to enduring, from early in development to late in life, and traverse spatial scales from protein modification to complex circuits. Integrating these varied levels of neural signaling into a coherent and predictive picture of brain function remains the formidable challenge for modern neuroscience.

\section{Biographies}

Michael Ehlers is George Barth Geller professor of Neurobiology at Duke University. His laboratory investigates the cell biological mechanisms underlying neuronal architecture and plasticity. His current research focuses on mechanisms for signaling and molecular plasticity at glutamatergic synapses in the mammalian brain and their role in brain development and circuit function.

Gina Turrigiano is a professor of Biology at Brandeis, where her work centers on understanding the plasticity mechanisms that allow cortical networks to undergo experience-dependent refinement. 